\title{
Neutrophil-lymphocyte ratio predicts short-term mortality in patients hospitalized for acute decompensation of cirrhosis
}

\author{
Claudia MACCALI, Fernanda Cristina de AUGUSTINHO, Tamara Liana ZOCCHE, Telma Erotides SILVA, \\ Janaína Luz NARCISO-SCHIAVON and Leonardo de Lucca SCHIAVON
}

Received:17 March 2020

Accepted: 16 November 2020

\begin{abstract}
Background - Individuals with cirrhosis have a chronic systemic inflammation associated with an immune dysfunction, affecting the progression of the liver disease. The neutrophil-lymphocyte ratio (NLR) was proposed as a marker of systemic inflammatory response and survival in patients with cirrhosis. Objective - Evaluate the prognostic role of NLR in cirrhotic patients and its relation with inflammatory cytokines (IL-6, IL-10 and IL-17). Methods - In this prospective study two groups were evaluated: 1) Stable cirrhotic in outpatient follow-up (n=193); 2) Hospitalized cirrhotic for acute decompensation for at least 48 hours $(n=334)$ with admission and 48 hours tests evaluation. Circulating inflammatory cytokines were available for 130 hospitalized patients. Results - In outpatients with stable cirrhosis, NLR correlated with MELD score and other variables associated with severity of disease. However, after a median of 32 months of follow up NLR was not associated with mortality (HR 1.058, 95\%CI $0.900-1.243 ; P=0.495)$. In hospitalized patients, NLR at 48-hour after admission was independently associated with 90-day survival (HR $1.061,95 \%$ CI $1.020-1.103 ; P=0.003)$ in multivariate Cox-regression analysis. The 90 -day Kaplan-Meier survival probability was $87 \%$ for patients with a 48 -hour NLR $<3.6$ and $62 \%$ for NLR $\geq 3.6(P<0.001)$. Elevation of NLR in the first 48 hours was also independently associated with mortality (HR 2.038, 95\%CI 1295-3207; $P=0.002$ ). The 90-day Kaplan-Meier survival probability was $83 \%$ when NLR did not increase and $62 \%$ when NLR increased $(P<0.001)$. IL-6, IL-10 and IL-17 at admission were positively correlated with both admission and 48-hour NLR. Lower levels of baseline IL-10 were associated with NLR increase during first 48-hour. Conclusion - NLR evaluated at 48 hours of hospitalization and its early increase after admission were independently associated with short-term mortality in patients hospitalized for acute decompensation of cirrhosis.
\end{abstract}

Keywords - Liver cirrhosis; cytokines; prognosis; inflammation.

\section{INTRODUCTION}

The natural history of cirrhosis is usually characterized by an initial phase of compensated disease with relatively good prognosis, followed by an advanced phase, which gradually or abruptly progresses to complications, secondary to portal hypertension and/or hepatic insufficiency ${ }^{(1,2)}$. One of the consequences of cirrhosis is an immune system dysfunction that affects both innate and adaptive responses, and is associated with systemic inflammation and immunodeficiency. The chronic inflammatory state observed in these patients results from activation of immune system cells by bacterial translocation, with consequent endotoxemia and increased production of inflammatory cytokines ${ }^{(3)}$. Persistence of systemic inflammation is associated with disease progression, emergence of complications and worse prognosis ${ }^{(4)}$.

Parameters routinely used in cirrhotic patients for systemic inflammation evaluation have several limitations. Systemic inflammatory response syndrome criteria was previously associated with higher mortality in patients with acute-on-chronic liver failure (ACLF) and decompensated cirrhosis, however it may be compromised by hypersplenism, hyperventilation secondary to hepatic encephalopathy and use of beta blockers ${ }^{(1)}$. Total leukocyte count is used as a predictive factor of occurrence and prognosis of ACLF, but it may be underestimated due to hypersplenism ${ }^{(1,2)}$. Acute phase serum markers, such as C-reactive protein (CRP) and procalcitonin levels, are more accurate than systemic inflammatory response syndrome for the identification of systemic inflammation $^{(1,5)}$. However, CRP is synthetized by the liver and, therefore, its levels may be influenced by the degree of liver dysfunction ${ }^{(6)}$. In addition, although procalcitonin is a promising biomarker, data is still limited and it is not yet widely available ${ }^{(7)}$.

The neutrophil-lymphocyte ratio (NLR) is a systemic inflammatory response marker that assesses two distinct pathways of the immune system: systemic inflammation, by neutrophil count, and the immune regulation pathway represented by lymphocytes ${ }^{(8)}$. NLR has already been evaluated as a prognostic factor for coronary disease and malignancies ${ }^{(9-11)}$. In liver diseases, it was studied in several diseases such as nonalcoholic fatty liver disease, chronic hepatitis B, liver transplantation, hepatocellular carcinoma, and liver cirrhosis ${ }^{(12-18)}$. Nevertheless, data on the applicability of NLR as a prognostic marker in cirrhosis is scarce, especially among patients hospitalized for acute decompensation. The aim of this study was to evaluate the role of NLR as a prognostic marker in cirrhotic patients undergoing outpatient care and hospitalized for acute decompensation. Relationships between NLR and inflammatory cytokines were also studied in a subset of hospitalized patients. 


\section{METHODS}

\section{Patients}

This study is part of a project that aims to follow two cohorts of adult patients ( $\geq 18$ years of age) with liver cirrhosis followed at the University Hospital of the Federal University of Santa Catarina. Details about those cohorts were previously published ${ }^{(19-20)}$ and are briefly presented below. Diagnosis of cirrhosis was histologically defined (when available) or by combining clinical, imaging, and laboratory findings in the presence of portal hypertension. The first cohort included 193 patients with stable cirrhosis attended in the Outpatient Clinic. Exclusion criteria in this group were diagnosis of hepatocellular carcinoma, diagnosis of bacterial infections during the last 7 days, interferon-based therapy during the last 30 days, or refusal or inability of the patient to understand the terms of the informed consent. The second cohort comprised 334 patients admitted to the emergency room due to acute decompensation of liver cirrhosis. Exclusion criteria were hospitalization for elective procedures or unrelated to complications of liver cirrhosis, hospitalization for less than 48 hours, absence of laboratory tests, and hepatocellular carcinoma outside Milan criteria.

The study protocol is in accordance with the ethical principles of the Declaration of Helsinki and it was approved by the Research Ethics Committee of the Federal University of Santa Catarina. Written informed consent was obtained from all patients or their legal guardians prior to the evaluation.

\section{Study design}

In the outpatient cohort, all patients were initially evaluated from June to October 2012. Patients were submitted to annual evaluation until 2014, with pre-defined visits, and the presence of complications, death or liver transplantation was assessed by periodic phone calls or during routine visits. The second cohort was comprised of patients hospitalized for acute decompensation of cirrhosis between January 2011 and November 2016. Acute decompensation was defined as the acute development of hepatic encephalopathy, large ascites, gastrointestinal bleeding, bacterial infection, or any combination of these ${ }^{(21)}$. Patients were monitored during hospitalization and 30- and 90-day mortality was assessed by a phone call. In the case of more than one hospital admission during the study period, only the first hospitalization was considered.

Patients were considered active drinkers if they reported an average overall consumption of 21 or more drinks per week for men and 14 or more drinks per week for women during the 4 weeks before enrollment (one standard drink is equal to $12 \mathrm{~g}$ absolute alcohol) (22).

All patients admitted for acute decompensation of cirrhosis in our institution are actively screened for bacterial infections. A diagnostic paracentesis was performed in all patients with ascites at admission. Spontaneous bacterial peritonitis (SBP) was diagnosed when the neutrophil count of the ascitic fluid was $\geq 250$ neutrophils $/ \mathrm{mm}^{3}$ in the absence of intra-abdominal source of infection, regardless of negative culture ${ }^{(23)}$. Criteria for diagnosing other infections than SBP were adapted from Centers for Disease Control and Prevention ${ }^{(24)}$. Hepatic encephalopathy was graded using the West-Haven criteria ${ }^{(25)}$, and precipitating factors were investigated while lactulose was initiated and adjusted as needed. Patients with acute variceal bleeding received intravenous octreo- tide, prophylactic antibiotic and underwent emergency endoscopic treatment after clinical stabilization.

The liver disease severity was assessed by Child-Pugh score and Model for End-Stage Liver Disease (MELD) ${ }^{(26)}$. The definitions of ACLF and CLIF-SOFA (chronic liver failure - sequential organ failure assessment) were based on the EASL-CLIF Consortium ${ }^{(21)}$.

NLR was calculated by dividing the absolute neutrophil count by the absolute lymphocyte count. NLR was calculated based on the initial laboratory tests in 2012 in the outpatients group, and on admission and after 48 hours in hospitalized patients.

\section{Circulating cytokine measurements}

The cytokines IL-6, IL-10 and IL-17 were measured in samples collected at the hospital admission from February 2012 to August 2014 of a subgroup of 130 individuals from the inpatient cohort. Data specifically related to this cohort were previously published ${ }^{(20)}$. The quantitative determination of IL-6, IL-10, and IL-17A in the serum was performed using a cytometric bead array (CBA) kit (BD Biosciences; San Diego, CA). The fluorescence produced by the CBA beads was measured on a FASCSVerse Flow Cytometer (BD FACSVerseTM, San Jose, CA, USA) and analyzed using Software FCAP Array 3.0 (BD Biosciences). Detection limits were $2.4 \mathrm{pg} / \mathrm{mL}$ for IL-6, $4.5 \mathrm{pg} / \mathrm{mL}$ for IL-10, and $18.9 \mathrm{pg} / \mathrm{mL}$ for IL-17A.

\section{Statistical analysis}

Normality of the variable distribution was determined using the Kolmogorov-Smirnov test. The correlation between the numerical variables was evaluated using Spearman's correlation coefficient. Continuous variables were compared using the MannWhitney test. Univariate and multivariate Cox regression analyses (enter method) were used to investigate the association between the variables and survival. The best cutoff point for predicting mortality was chosen based on the ROC curve. Kaplan-Meier curves were used to illustrate survival according to two strata. All tests were performed by the SPSS software, version 17.0 (SPSS, Chicago, IL, USA). A $P$ value of less than 0.05 was considered statistically significant.

\section{RESULTS}

\section{Characteristics of included patients}

The study included 513 individuals, 193 with stable cirrhosis and 320 hospitalized for acute decompensation. TABLE 1 shows the characteristics of the patients included. In the cohort of stable cirrhosis, mean age was $54.4 \pm 12.5$ years and subjects were predominantly male $(67.9 \%)$. The most common etiology of cirrhosis was alcohol abuse (53.9\%), followed by hepatitis C (32.6\%). Previous history of cirrhosis decompensation was observed in $73.6 \%$ of the sample and only $5.7 \%$ of the individuals reported active alcoholism. The mean MELD score was $10.10 \pm 2.51$ and $63.7 \%$ of the individuals were classified as Child-Pugh A.

In the group of patients hospitalized for acute decompensation, the mean age was $55.7 \pm 10.7$ years and $73.4 \%$ were male. Previous decompensation was reported by $66.2 \%$ of the patients and active alcoholism was present in $31.9 \%$. At admission, upper gastrointestinal bleeding was observed in $40.0 \%$ of cases, ascites in $52.2 \%$, hepatic encephalopathy in $49.4 \%$, bacterial infections in $44.7 \%$ and ACLF in $24.4 \%$. The mean MELD score was $16.81 \pm 6.36$ and $43.4 \%$ of the subjects were classified as Child-Pugh C. 
TABLE 1. Characteristics of included patients.

\begin{tabular}{|c|c|c|}
\hline Variables & $\begin{array}{l}\text { Stable } \\
\text { cirrhosis } \\
(\mathrm{n}=193) \\
\end{array}$ & $\begin{array}{c}\text { Acute } \\
\text { decompensation } \\
(\mathrm{n}=320)\end{array}$ \\
\hline Age (years), mean $\pm \mathrm{SD}$ & $54.36 \pm 12.48$ & $55.67 \pm 10.77$ \\
\hline Male gender, n (\%) & $131(67.9)$ & $235(73.4)$ \\
\hline \multicolumn{3}{|l|}{ Etiology of cirrhosis, n (\%) } \\
\hline Alcohol & $104(53.9)$ & $182(56.9)$ \\
\hline Hepatitis C & $63(32.6)$ & $114(35.6)$ \\
\hline Hepatitis B & $11(5.7)$ & $19(6.0)$ \\
\hline Cryptogenic & $20(10.4)$ & $25(7.8)$ \\
\hline NAFLD & $7(3.6)$ & $20(6.3)$ \\
\hline Autoimmune & $8(4.1)$ & $10(3.1)$ \\
\hline Previous decompensation, $\mathrm{n}(\%)$ & $142(73.6)$ & $210(66.2)$ \\
\hline Active alcoholism, n (\%) & $11(5.7)$ & $102(31.9)$ \\
\hline Hepatocellular carcinoma & 0 & $21(7.2)$ \\
\hline Esophageal varices & $155(81.2)$ & $83(74.1)^{\#}$ \\
\hline Propranolol, n (\%) & $110(57.0)$ & $102(32.1)$ \\
\hline \multicolumn{3}{|l|}{ Complications in the evaluation, $\mathrm{n}(\%)$} \\
\hline Ascites & $43(22.3)$ & $167(52.2)$ \\
\hline Hepatic encephalopathy & $20(10.4)$ & $158(49.4)$ \\
\hline Gastrointestinal bleeding & 0 & $128(40.0)$ \\
\hline Bacterial infection & 0 & $143(44.7)$ \\
\hline ACLF & NA & $78(24.4)$ \\
\hline \multicolumn{3}{|l|}{ Laboratory data } \\
\hline Leukocyte Count (x $\left.10^{9}\right)$, median & 4.78 & 7.51 \\
\hline Sodium $(\mathrm{meq} / \mathrm{L})$, mean $\pm \mathrm{SD}$ & $137.41 \pm 2.65$ & $135.48 \pm 5.16$ \\
\hline Creatinine $(\mathrm{mg} / \mathrm{dL})$, median & 0.90 & 1.10 \\
\hline INR, median & 1.20 & 1.44 \\
\hline Albumin $(\mathrm{g} / \mathrm{dL})$, mean $\pm \mathrm{SD}$ & $3.41 \pm 0.49$ & $2.47 \pm 1.84$ \\
\hline $\mathrm{CRP}(\mathrm{mg} / \mathrm{L})$, median & 3.50 & 15.10 \\
\hline Total bilirubin $(\mathrm{mg} / \mathrm{dL})$, median & 1.00 & 2.10 \\
\hline NLR, median & 2.10 & 4.05 \\
\hline \multicolumn{3}{|l|}{ Child-Pugh classification, n (\%) } \\
\hline A & $123(63.7)$ & $24(7.5)$ \\
\hline $\mathrm{B}$ & $59(30.6)$ & $156(49.1)$ \\
\hline $\mathrm{C}$ & $11(5.7)$ & $138(43.4)$ \\
\hline MELD, mean + SD & $10.10 \pm 2.51$ & $16.81 \pm 6.36$ \\
\hline CLIF-SOFA, mean \pm SD & NA & $6.45 \pm 2.62$ \\
\hline
\end{tabular}

"Information available for 112 patients.

SD: standard deviation; NAFLD: non-alcoholic fatty liver disease; AIH: autoimmune hepatitis; ACLF: acute-on-chronic liver failure; INR: international normalized ratio; CRP: C-reactive protein; NLR: neutrophil to lymphocyte ratio; MELD: Model for End-stage Liver Disease; CLIF-SOFA: chronic liver failure - sequential organ failure assessment.

\section{NLR in outpatients with stable cirrhosis}

In patients with stable cirrhosis, NLR was positively correlated with total bilirubin levels $(\mathrm{r}=0.183, P=0.011)$, INR $(\mathrm{r}=0.151$, $P=0.037)$, CRP $(\mathrm{r}=0.218, P=0.002)$, and MELD $(\mathrm{r}=0.224$, $P=0.002)$. No significant correlation was observed between the other numerical variables studied and NLR. Presence of previous decompensation was associated with higher NLR values (2.65 vs $2.03, P=0.012$ ). There was no association between NLR and other categorical variables.

The median follow-up was 32 months in the cohort of stable cirrhosis. During the studied period, $35(19 \%)$ patients died and $18(10 \%)$ were submitted to liver transplantation. There was no relationship between NLR and transplant-free survival in patients with stable cirrhosis (HR 1.058, 95\% CI 0.900-1.243, $P=0.495$ ) in univariate cox regression.

\section{NLR in patients hospitalized for acute decompensation of cirrhosis}

In the cohort of hospitalized patients, NLR at admission was positively correlated with creatinine $(\mathrm{r}=0.166, P=0.003)$, total bilirubin ( $\mathrm{r}=0.168, P=0.003)$, INR ( $\mathrm{r}=0.115, P=0.040)$, CRP $(\mathrm{r}=0.364, P=0.001)$, CLIF-SOFA ( $\mathrm{r}=0.195, P=0.001)$, Child-Pugh $(\mathrm{r}=0.163, P=0.004)$, and MELD $(\mathrm{r}=0.235 ; P<0.001)$; and negatively correlated with albumin $(\mathrm{r}=-0.176, P=0.002)$, sodium $(\mathrm{r}=-0.272$, $P<0.001)$, and mean arterial pressure (MAP) $(\mathrm{r}=-0.164, P=0.003)$.

Higher NLR values were observed in the case of alcoholic etiology of cirrhosis (4.86 vs $3.64, P=0.003$ ), ACLF (6.36 vs 3.65 , $P<0.001)$, Child-Pugh C (4.94 vs $3.63, P=0.003$ ), and bacterial infection (4.95 vs 3.49, $P=0.001$ ); lower values were observed in those with hepatitis $C(3.32$ vs $4.57, P=0.027)$ and among patients with hepatocellular carcinoma ( 2.41 vs $4.01, P=0.002$ ). There were no associations between NLR and the other categorical variables, including previous decompensation (4.11 vs $3.88, P=0.663$ ).

\section{NLR and prognosis of patients hospitalized for acute decompensation}

Univariate Cox regression was performed to investigate factors associated with survival among patients with acute decompensation of cirrhosis (TABLE 2). Ninety-day mortality was associated with complications of cirrhosis such as ascites, hepatic encephalopathy, bacterial infections and ACLF. Nevertheless, gastrointestinal bleeding was significantly more frequent in survivors. Patients who died also had higher median leukocytes, creatinine, INR, CRP and total bilirubin, as well as lower mean sodium and albumin levels. A higher proportion of patients classified as Child-Pugh C, and with a higher MELD and CLIF-SOFA scores died during followup. Higher median NLR on admission was also associated with a worse outcome in the univariate Cox regression analysis (4.68 vs 3.91, HR=1.047, $P<0.001)$. Similar results were obtained for the NLR evaluated after 48 hours of hospitalization ( 4.60 vs 2.68 , HR $1.111, P<0.001)$. Previous decompensation showed no impact in 90-day survival.

Multivariate Cox regression analysis was performed including the following variables: admission NLR, ACLF, Child-Pugh C, and MELD. Other variables already included in the prognostic models were not included in the multivariate analysis to avoid collinearity and to keep an acceptable number of events per variable. In this analysis, ACLF (HR 2.007, CI 95\% 1.094-3.680, $P=0.024$ ), ChildPugh C (HR 2.213, CI 95\% 1.211-4.046, $P=0.010$ ) and MELD (HR 1.048, CI 95\% 1.000-1.098; $P=0.049$ ) were associated with lower survival. Admission NLR was not an independent prognostic factor (HR 1.022, CI 95\% 0.987-1.057, $P=0.221$ ). A subsequent analysis was performed including NLR after 48 hours and the same variables mentioned previously. In this analysis, ACLF (HR 1.869 , CI 95\% 1.024-3.411, $P=0.042$ ), Child-Pugh C (HR 2.122, CI $95 \% 1.154-3.904, P=0.015$ ) and 48-hour NLR (HR 1.061, CI 95\% $1.020-1.103, P=0.003)$ were independent predictors of survival. The area under the ROC curve for 48-hour NLR predicted mortality at 90 days was 0.691 (CI 95\% 0.621-0.762). The best cutoff point chosen by the ROC curve for 48 -hour NLR predicted mortality at 90 days was 3.6. The Kaplan-Meier survival probability at 90 days was $87 \%$ for patients with 48 -hour NLR $<3.6$ and $62 \%$ for NLR $\geq 3.6$ 
TABLE 2. Factors associated with 90-day mortality in patients hospitalized for acute decompensation of cirrhosis.

\begin{tabular}{|c|c|c|c|c|}
\hline Variables & $\begin{array}{c}\text { Survivors } \\
(\mathrm{n}=243)\end{array}$ & $\begin{array}{c}\text { Non-survivors } \\
(\mathrm{n}=77)\end{array}$ & HR (CI 95\%) & $P$ \\
\hline Male gender, n (\%) & $180(74.1)$ & $55(71.4)$ & $0.903(0.551-1.480)$ & 0.685 \\
\hline Alcohol & $138(56.8)$ & $44(57.1)$ & $1.021(0.650-1.603)$ & 0.929 \\
\hline Hepatitis C & $89(36.6)$ & $25(32.5)$ & $0.838(0.520-1.350)$ & 0.467 \\
\hline Hepatitis B & $13(5.4)$ & $6(7.8)$ & $1.385(0.602-3.186)$ & 0.444 \\
\hline Active alcoholism, n (\%) & $54(24.8)$ & $23(22.5)$ & $0.898(0.551-1.463)$ & 0.666 \\
\hline Previous decompensation, $\mathrm{n}(\%)$ & $160(65.8)$ & $50(64.9)$ & $0.951(0.596-1.519)$ & 0.834 \\
\hline \multicolumn{5}{|l|}{ Complication in admission, $\mathrm{n}(\%)$} \\
\hline Ascites & $108(44.4)$ & $59(76.6)$ & $3.545(2.090-6.012)$ & $<0.001$ \\
\hline Hepatic encephalopathy & $110(45.3)$ & $48(62.3)$ & $1.822(1.149-2.889)$ & 0.011 \\
\hline Gastrointestinal Bleeding & $106(43.6)$ & $22(28.6)$ & $0.573(0.349-0.939)$ & 0.027 \\
\hline \multicolumn{5}{|l|}{ Laboratory data } \\
\hline Leukocyte count (x109), median & 7.00 & 7.25 & $1.045(1.008-1.083)$ & 0.017 \\
\hline Sodium $(\mathrm{meq} / \mathrm{L})$, mean $\pm \mathrm{SD}$ & $135.97 \pm 4.88$ & $133.96 \pm 5.74$ & $0.938(0.901-0.976)$ & 0.002 \\
\hline INR, median & 1.40 & 1.65 & $1.829(1.462-2.288)$ & $<0.001$ \\
\hline Albumin $(\mathrm{g} / \mathrm{dL})$, mean $\pm \mathrm{SD}$ & $2.40 \pm 0.61$ & $2.00 \pm 0.55$ & $0.256(0.165-0.399)$ & $<0.001$ \\
\hline $\mathrm{CRP}(\mathrm{mg} / \mathrm{L})$, median & 10.90 & 28.30 & $1.007(1.004-1.010)$ & $<0.001$ \\
\hline Bilirubin total $(\mathrm{mg} / \mathrm{dL})$, median & 1.80 & 3.15 & $1.049(1.022-1.077)$ & $<0.001$ \\
\hline Admission NLR, median & 3.91 & 4.68 & $1.047(1.021-1.073)$ & $<0.001$ \\
\hline Child-Pugh C, n (\%) & $83(34.3)$ & $55(72.4)$ & $4.013(2.426-6.638)$ & $<0.001$ \\
\hline MELD score, mean \pm SD & $14.35 \pm 5.00$ & $22.44 \pm 6.95$ & $1.116(1.087-1.146)$ & $<0.001$ \\
\hline CLIF-SOFA, mean \pm SD & $5.83 \pm 2.30$ & $8.52 \pm 2.57$ & $1.393(1.290-1.503)$ & $<0.001$ \\
\hline \multicolumn{5}{|l|}{ Vital signs } \\
\hline
\end{tabular}

SD: standard deviation; ACLF: acute-on-chronic liver failure; INR: international normalized ratio; CPR: C-reactive protein; NLR: neutrophil-lymphocyte ratio; MELD: Model for End-stage Liver Disease; CLIF-SOFA: chronic liver failure - sequential organ failure assessment. MAP: mean arterial pressure; SpO2 / FiO2: oxygen saturation / inspired oxygen fraction ratio.

$(P<0.001)($ FIGURE 1). At the cutoff of 3.6, 48-hour NLR showed a sensitivity of $69 \%$, specificity of $65 \%$, positive predictive value (PPV) of $38 \%$ and a negative predictive value (NPV) of $87 \%$ for predicting 90-day mortality.

Prognostic ability of 48-hour NLR was not significantly influenced by the history of previous decompensation. Among those without previous decompensation, the survival probability was $87.5 \%$ for patients with 48 -hour NLR $<3.6$ and $63.0 \%$ for those with greater values $(P=0.003)$. In this case, 48 -hour NLR showed a sensitivity of $74 \%$, specificity of $59 \%$, PPV of $37 \%$ and NPV of $88 \%$. Similarly, for subjects with previous history of hepatic decompensation, 90 -day survival was $86.5 \%$ for NLR $<3.6$ and $60.7 \%$ for the remaining individuals $(P<0.001)$. NLR exhibited sensitivity of $66 \%$, specificity of $68 \%$, PPV of $39 \%$ and NPV of $87 \%$.

\section{Dynamic NLR changes in the first 48 hours and prognosis in hospitalized patients with cirrhosis}

When dynamic changes of NLR during the first 48 hours of hospitalization were evaluated, a higher proportion of patients who died exhibited NLR increase when compared to survivors $(51 \%$ vs $26 \%, P<0.001)$. This increment in NLR was associated with

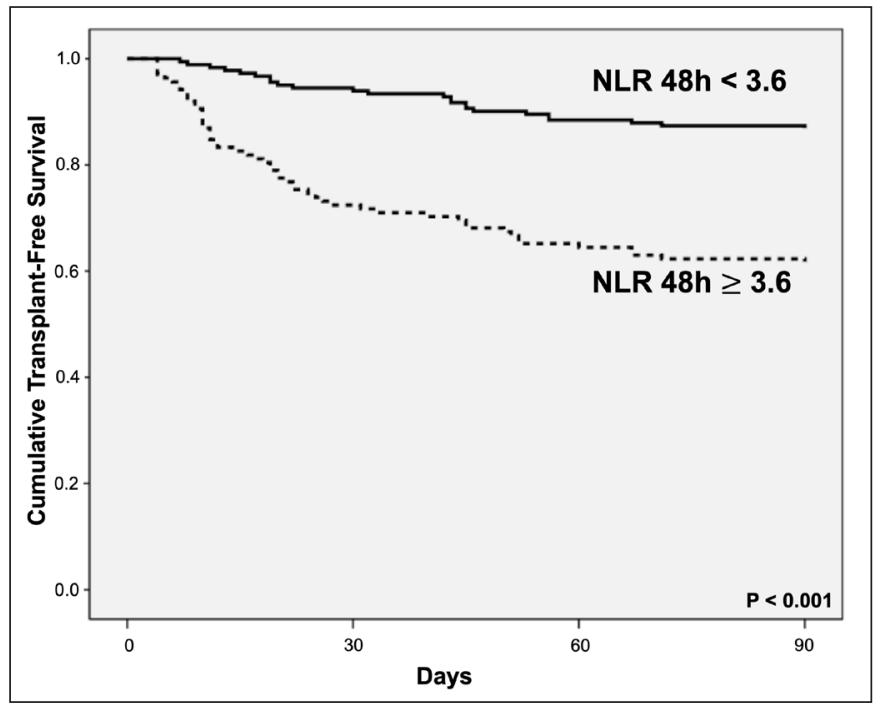

FIGURE 1. Kaplan-Meier curve cumulative survival at 90 days according to the 48-hour NLR. 
90-day mortality in univariate Cox regression analysis (HR 2.488, CI 95\% 1.591-3.891, $P<0.001)$. In the multivariate analysis, presence of ACLF (HR 1.870, CI 95\% 1.021-3.423, $P=0.043$ ), ChildPugh C (HR 2,088, CI 95\% 1.138-3.834, $P=0.018$ ), MELD (HR 1.057 , CI $95 \% 1.010-1.105, P=0.017)$, and increase in NLR in the first 48 hours were independent predictors of 90-day mortality (HR 2.038, CI 95\% 1.295-3.207, $P=0.002$ ). The 90-day Kaplan-Meier survival probability was $83 \%$ in cases with no increase in NLR and $62 \%$ in those with increase in NLR $(P<0.001)$ (FIGURE 2). Increase in NLR showed a sensitivity of $51 \%$, specificity of $74 \%$, PPV of $38 \%$ and NPV of $83 \%$.

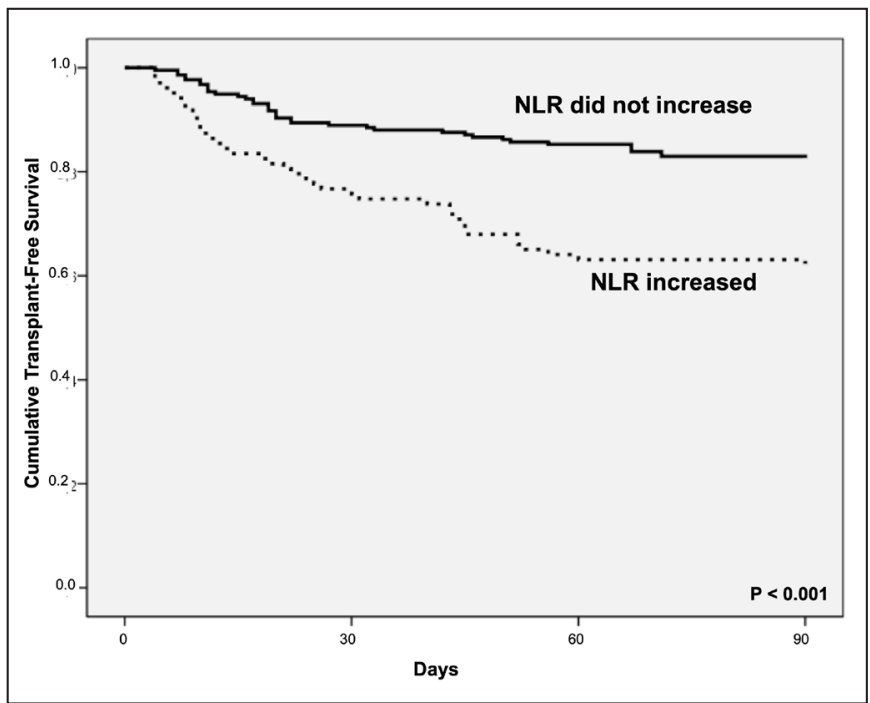

FIGURE 2. Kaplan-Meier curve survival probability at 90 days according to the occurrence or non-occurrence of NLR elevation in the first 48 hours.

The prognostic impact of NLR increase was evaluated according to history of previous decompensation. For patients without previous decompensation, the survival probability was $80 \%$ in cases with no increase in NLR and $67 \%$ in those with increase in NLR $(P=0.127)$. In these case, increase in NLR showed a sensitivity of $48 \%$, specificity of $68 \%$, PPV of $33 \%$ and NPV of $80 \%$. For those with previous decompensation, the survival probability was $84 \%$ in cases with no increase in NLR and 59\% in those with increase in NLR $(P<0.001)$. Increase NLR exhibited 52\% sensitivity, $78 \%$ specificity, $41 \%$ PPV and $84 \%$ NPV.

\section{NLR and inflammatory cytokines in acute decompensation of cirrhosis}

Circulating levels of inflammatory cytokines at admission were available for 130 patients hospitalized for acute decompensation of cirrhosis. Median IL-6, IL-17 and IL-10 were $19.30 \mathrm{pg} / \mathrm{mL}, 3.42$ $\mathrm{pg} / \mathrm{mL}$, and $2.24 \mathrm{pg} / \mathrm{mL}$, respectively. IL-6 was positively correlated with admission $(\mathrm{r}=0.438, P<0.001)$ and 48 -hour NLR $(\mathrm{r}=0.319$, $P<0.001)$. No differences were observed in IL- 6 concentrations among those with or without increase in NLR in the first 48 hours (15.90 vs $18.42 ; P=0.876)$. Similarly, IL-17 at admission correlated with admission NLR ( $\mathrm{r}=0.195 ; P=0.027)$ and, most strongly, with 48-hour NLR $(r=0.350 ; P<0.001)$. However, once again, differences in IL-17 levels were not noticed when compared those who exhibited NLR increase with those who don't $(P=0.311)$. Circulating IL-10 were correlated with NLR of both admission $(\mathrm{r}=0.391, P<0.001)$ and 48 hours ( $\mathrm{r}=0.199 ; P=0.032)$. Interestingly, lower median IL10 levels were observed among those who showed an increase in NLR in the first 48 hours of hospitalization $(2.00 \mathrm{pg} / \mathrm{mL}$ vs 2.75 $\mathrm{pg} / \mathrm{mL}, P=0.028)$.

\section{DISCUSSION}

In liver cirrhosis, there is a persistent systemic inflammation due to deregulation between pro and anti-inflammatory pathways, implying progression of liver disease and increased risk of decompensation ${ }^{(4)}$. Currently available tools for the evaluation of this inflammatory state have the potential to guide decisions regarding therapeutic options, follow-up and prognostic evaluation, however, they have important limitations ${ }^{(1)}$. In this study, the NLR was evaluated as a marker of systemic inflammatory response in patients with liver cirrhosis followed on an outpatient basis, and in patients hospitalized for acute decompensation.

In outpatients with stable cirrhosis, NLR was not associated with survival after a median 32 months of follow-up. In 2012, Biyik et al. demonstrated that NLR was an independent predictor of mortality in stable cirrhosis after long-term outpatient follow-up ${ }^{(17)}$. Patients with NLR greater than 2.72 had significantly higher mortality ${ }^{(17)}$. Other studies including patients listed for liver transplantation have shown that NLR is a useful tool to determine prognosis and outcome among subjects with low MELD and may aid in the decision of prioritize these patients for transplant, consider living donor or extension of donor criteria ${ }^{(14,18)}$. As in the present study, Leithead et al. found a positive correlation between NLR and INR, bilirubin and MELD, but in their results NLR presented as an independent survival factor ${ }^{(14)}$. When comparing the characteristics of patients included in the present study with other previously published cohorts, those included here had lower proportion of ascites, were younger, and mostly Child-Pugh A with low MELD scores. These findings suggest that, in outpatients with stable cirrhosis, NLR might perform better in the setting of more advanced liver disease.

In patients hospitalized for acute decompensation of cirrhosis, NLR presented a positive correlation with well-studied prognostic scores, such as CLIF-SOFA, Child-Pugh and MELD ${ }^{(27)}$. In addition, patients with Child-Pugh $\mathrm{C}$ and ACLF presented higher values of NLR, indicating that NLR is related to the severity of liver disease ${ }^{(8,28)}$. Hospitalized patients with infection also exhibited significantly higher NLR values. Similar to these results, Lliaz et al. demonstrated higher NLR values in patients with cirrhosis diagnosed with spontaneous bacterial peritonitis ${ }^{(29)}$. A retrospective Chinese study identified that the NLR assessed at admission was an independent predictor of hospital-acquired bacterial infections ${ }^{(30)}$. The higher NLR values in the presence of infection are probably due to the fact that immune system dysregulation and malnutrition are important risk factors for infection. Systemic inflammation associated with immune dysfunction and infection triggers an increase in neutrophils, which inhibit activation and induce lymphocyte apoptosis. This factor, associated with lymphopenia secondary to a state of chronic malnutrition, leads to a tendency of higher NLR values ${ }^{(8,30)}$.

Lower NLR values were observed among patients with hepatocellular carcinoma. Previous studies associated NLR with tumor staging in hepatocellular carcinoma, with higher values observed in individuals with more advanced disease ${ }^{(15,16)}$. However, in the present study, advanced hepatocellular carcinoma, outside the 
Milan criteria, was considered exclusion criterion. Thus, it is likely that hepatocellular carcinoma did not significantly affect the NLR values in this group and that the difference observed here is probably due to a less advanced baseline liver disease among patients hospitalized with hepatocellular carcinoma.

Although admission NLR was related with 90-day mortality in univariate Cox regression analysis, this association was not maintained in the multivariate analysis including other important prognostic factors. On the other hand, NLR assessed after 48 hours of hospitalization and increase in NLR during the first 48 hours of hospitalization were independently associated with survival. It is likely that these results reflect the ability of NLR to identify patients who presented with a more intense systemic inflammatory response as a result of a more severe decompensation or more advanced disease $^{(4)}$. In addition, NLR elevation during hospitalization suggests a more intense systemic inflammation and immune dysregulation, consequently inferring greater severity of the acute decompensation or an inadequate response to the treatment offered, impacting on mortality ${ }^{(31)}$. Previous studies investigating the relationship between NLR and early mortality (30 days) in different profiles of patients and applying different methodologies have suggested that higher NLR values are associated with higher mortality ${ }^{(8,31,32)}$. Recently, Rice and colleagues demonstrated that higher NLR values at hospital admission are related to lower survival at 90 days and one year, higher rates of hospital readmission in 90 days and longer hospital stay ${ }^{(33)}$. There are no previous studies evaluating the dynamic changes of NLR values during hospitalization of patients with cirrhosis.

History of previous decompensation was not associated with NLR values and was also not related to survival in patients with acute decompensation. In addition, previous decompensation exhibited no significant impact on prognostic ability of NLR assessed after 48 hours of hospitalization. In patients with history of past decompensation there was a slightly better performance of NLR increase for predicting mortality as compared to those without previous decompensation. However, the overall impact was numerically low, probably resulting from the reduction of sample size for this sub-analysis. Although the knowledge of previous complications of any patient is of unquestionable clinical relevance, recent studies showed that, in the setting of acute decompensation of cirrhosis, short-term prognosis appears to be more related to the severity of the acute episode than to previous complications ${ }^{(21,34)}$. On the other hand, history of previous acute decompensation episodes exhibited great impact on long-term prognosis in ACLF survivors ${ }^{(35)}$.

Another interesting finding from our study is that gastrointestinal bleeding was more frequent in the survivor group. Although variceal bleeding is a serious complication of cirrhosis, it has been associated with improved survival and lower organ failure scores in patients admitted in intensive care units ${ }^{(36,37)}$. In addition, our institution has an established protocol for treating acute variceal bleeding, which contributes to better outcomes in these cases. Therefore, when compared to other complications presented at hospital admission, particularly bacterial infection and renal dysfunction, variceal bleeding is generally related to a better prognosis.

IL-6, IL-10 and IL-17 correlated positively with admission and 48-hour NLR. Cirrhotic patients have increased levels of inflammatory and anti-inflammatory cytokines, resulting in a state of chronic inflammation ${ }^{(38)}$. In a previous published analysis of this same cohort conducted by our group, an increase in these cytokines was observed both in patients with compensated and decompensated cirrhosis, with higher values in the case of acute events ${ }^{(20)}$. A study conducted in China also evaluated the association between NLR and cytokines, finding a moderate correlation between NLR and IL-6 and IL- ${ }^{(32)}$. Lower median IL-10 was observed in patients with increase in NLR in the first 48 hours of hospitalization. IL-10 is an important anti-inflammatory cytokine that is increased in liver cirrhosis as a compensatory response to the chronic inflammatory state and endotoxemia ${ }^{(20,39)}$. It is possible that a less intense basal anti-inflammatory response, demonstrated here by lower levels of IL-10, allows a progression to a more robust subsequent inflammatory response, resulting in a more pronounced NLR increase.

In conclusion, NLR evaluated after 48 hours of hospitalization and increase in NLR during the first 48 hours of admission were independently associated with short-term mortality in patients hospitalized for acute decompensation of cirrhosis. NLR is a simple, inexpensive, widely available, and easy to apply tool that can contribute for a better prognostic assessment in patients with advanced liver cirrhosis.

\section{ACKNOWLEDGEMENTS}

We would like to thank all other collaborators who participated in this study: Josiane Fischer, Camila Matiollo, Elayne Cristina de Morais Rateke, Tania Silva Frode, Silvana Vigil de Mello, Julia Rosa and Esther Buzaglo Dantas-Correa.

\section{Authors' contribution}

Maccali C: writing of article and data recording. Augustinho FC, Zocche TL and Silva TE: data recording. Narciso-Schiavon JL: research execution and critical review of the manuscript. Schiavon LL: statistical analysis, research execution and critical review of the manuscript.

\section{Orcid}

Claudia Maccali: 0000-0002-0300-2066.

Fernanda Cristina de Augustinho: 0000-0003-3190-7177.

Tamara Liana Zocche: 0000-0002-7948-0953.

Telma Erotides Silva: 0000-0002-9808-5182.

Janaína Luz Narciso-Schiavon: 0000-0002-6228-4120.

Leonardo de Lucca Schiavon: 0000-0003-4340-6820. 
Maccali C, Augustinho FC, Zocche TL, Silva TE, Narciso-Schiavon JL, Schiavon LL. Razão neutrófilo-linfócito prediz mortalidade em curto prazo em pacientes hospitalizados com cirrose hepática descompensada. Arq Gastroenterol. 2021;58(2):131-8.

RESUMO - Contexto - Na cirrose há um quadro crônico de inflamação sistêmica associada a disfunção imune, que impactam na progressão da doença hepática. A razão neutrófilo-linfócito (RNL) foi proposta como um marcador de resposta inflamatória sistêmica e sobrevida em pacientes com cirrose hepática. Objetivo - Avaliar o papel de RNL como marcador prognóstico em portadores de cirrose hepática e sua relação com citocinas inflamatórias (IL-6, IL-10 e IL-17). Métodos -É um estudo prospectivo com duas coortes: 1) pacientes cirróticos estáveis em acompanhamento ambulatorial (n=193); 2) pacientes cirróticos hospitalizados por descompensação aguda por no mínimo 48 horas (n=334) com avaliação de exames de admissão de 48 horas. Citocinas inflamatórias séricas estavam disponíveis em 130 pacientes hospitalizados. Resultados - Nos pacientes ambulatoriais com cirrose estável, RNL se correlacionou com MELD e outras variáveis associadas com gravidade da doença. Entretanto, após uma mediana de 32 meses de seguimento, RNL não apresentou associação com mortalidade (HR 1.058, 95\% CI 0.900-1.243; $P=0.495$ ). Nos pacientes hospitalizados, RNL de 48 horas após a admissão apresentou associação independente com sobrevida em 90 dias (HR 1.061, 95\%CI 1.020-1.103; $P=0.003$ ) na regressão multivariada de Cox. A probabilidade de sobrevivência pela curva de Kaplan-Meier em 90 dias foi de $87 \%$ em pacientes com RNL de 48 horas $<3.6$ e $62 \%$ nos pacientes com RNL $\geq 3.6(P<0.001)$. A elevação de RNL nas primeiras 48 horas também foi um fator independente associado a mortalidade (HR 2.038 , $95 \%$ CI 1295-3207; $P=0.002$ ). A avaliação de sobrevida em 90 dias pela curva de Kaplan-Meier foi de $83 \%$ nos pacientes em que RNL não apresentou elevação e $62 \%$ nos que apresentaram elevação de RNL $(P<0.001)$. IL-6, IL-10 e IL-17 na admissão se correlacionaram positivamente com RNL de admissão e de 48 horas. Níveis mais baixos de IL-10 basal foram associados com elevação de RNL nas primeiras 48 horas. Conclusão - RNL avaliada em 48 horas de hospitalização e sua elevação precoce após a admissão foram fatores independentemente associados a mortalidade em curto prazo nos pacientes hospitalizados com descompensação aguda da cirrose.

Palavras-chave - Cirrose hepática; citocinas; prognóstico; inflamação.

\section{REFERENCES}

1. Dirchwolf M, Ruf AE. Role of systemic inflammation in cirrhosis: From pathogenesis to prognosis. World J Hepatol. 2015;7:1974-1981.

2. Jalan R, Gines P, Olson JC, Mookerjee RP, Moreau R, Garcia-Tsao G, et al. Acute-on chronic liver failure. J Hepatol. 2012;57:1336-1348.

3. Noor MT, Manoria P. Immune Dysfunction in Cirrhosis. J Clin Transl Hepatol. 2017;5:50-58.

4. Albillos A, Lario M, Alvarez-Mon M. Cirrhosis-associated immune dysfunction: distinctive features and clinical relevance. J Hepatol. 2014;61:1385-1396.

5. Lazzarotto C, Ronsoni MF, Fayad L, Nogueira CL, Bazzo ML, Narciso-Schiavon JL, et al. Acute phase proteins for the diagnosis of bacterial infection and prediction of mortality in acute complications of cirrhosis. Ann Hepatol. 2013; 12:599-607.

6. Pieri G, Agarwal B, Burroughs AK. C-reactive protein and bacterial infection in cirrhosis. Ann Gastroenterol. 2014;27:113-20.

7. Dong R, Wan B, Lin S, Wang M, Huang J, Wu Y, et al. Procalcitonin and Liver Disease: A Literature Review. J Clin Transl Hepatol. 2019;7:51-5.

8. Kwon JH, Jang JW, Kim YW, Lee SW, Nam SW, Jaegal D, et al. The usefulness of C-reactive protein and neutrophil-to-lymphocyte ratio for predicting the outcome in hospitalized patients with liver cirrhosis. BMC Gastroenterol. 2015;15:146-153.

9. Tamhane UU, Aneja S, Montgomery D, Rogers EK, Eagle KA, Gurm HS. Association between admission neutrophil to lymphocyte ratio and outcomes in patients with acute coronary syndrome. Am J Cardiol. 2008;102:653-7.

10. Halazun KJ, Aldoori A, Malik HZ, Al-Mukhtar A, Prasad KR, Toogood GJ, et al. Elevated preoperative neutrophil to lymphocyte ratio predicts survival following hepatic resection for colorectal liver metastases. Eur J Surg Oncol. 2008;34:55-60.

11. Zhao W, Wu Z, Li Y, Jia H, Chen M, Gu X, et al. Pretreatment neutrophil-to-lymphocyte ratio and its dynamic changes are associated with the overall survival in advanced cancer patients undergoing palliative care. Sci Rep. 2016;6:31394-404.

12. Alkhouri N, Morris-Stiff G, Campbell C, Lopez R, Tamimi TA, Yerian L, et al. Neutrophil to lymphocyte ratio: a new marker for predicting steatohepatitis and fibrosis in patients with nonalcoholic fatty liver disease. Liver Int. 2012;32:297-302.

13. Kekilli M, Tanoglu A, Sakin YS, Kurt M, Ocal S, Bagci S. Is the neutrophil to lymphocyte ratio associated with liver fibrosis in patients with chronic hepatitis B? World J Gastroenterol. 2015;21:5575-81.

14. Leithead JA, Rajoriya N, Gunson BK, Ferguson JW. Neutrophil-to-lymphocyte ratio predicts mortality in patients listed for liver transplantation. Liver Int. 2015;35:502-9.
15. Najjar M, Agrawal S, Emond JC, Halazun KJ. Pretreatment neutrophil-lymphocyte ratio: useful prognostic biomarker in hepatocellular carcinoma. J Hepatocell Carcinoma. 2018:5:17-28

16. Li SH, Wang QX, Yang ZY, Jiang W, Li C, Sun P, et al. Prognostic value of the neutrophil-to-lymphocyte ratio for hepatocellular carcinoma patients with portal/hepatic vein tumor thrombosis. World J Gastroenterol. 2017;23: 3122-32.

17. Biyik M, Ucar R, Solak Y, Gungor G, Polat I, Gaipov A, et al. Blood neutrophil-to-lymphocyte ratio independently predicts survival in patients with liver cirrhosis. Eur J Gastroenterol Hepatol. 2013;25:435-41.

18. Kalra A, Wedd JP, Bambha KM, Gralla J, Golden-Mason L, Collins C, et al. Neutrophil-to-lymphocyte ratio correlates with proinflammatory neutrophils and predicts death in low model for end-stage liver disease patients with cirrhosis. Liver Transpl. 2017;23:155-165.

19. Correa CG, Colombo Bda S, Ronsoni MF, Soares ESPE, Fayad L, Silva TE, et al. Circulating insulin-like growth factor-binding protein 3 as prognostic biomarker in liver cirrhosis. World J Hepatol. 2016;8:739-48.

20. Fischer J, Silva TE, Soares ESPE, Colombo BS, Silva MC, Wildner LM, et al. From stable disease to acute-on-chronic liver failure: Circulating cytokines are related to prognosis in different stages of cirrhosis. Cytokine. 2017;91:162-9.

21. Moreau R, Jalan R, Gines P, Pavesi M, Angeli P, Cordoba J, et al. Acute-onchronic liver failure is a distinct syndrome that develops in patients with acute decompensation of cirrhosis. Gastroenterology. 2013;144:1426-37.

22. Addolorato G, Leggio L, Ferrulli A, Cardone S, Vonghia L, Mirijello A, et al. Effectiveness and safety of baclofen for maintenance of alcohol abstinence in alcohol-dependent patients with liver cirrhosis: randomised, double-blind controlled study. Lancet. 2007;370:1915-22.

23. European Association for the Study of the Liver. Clinical Practice Guidelines for the management of patients with decompensated cirrhosis. J Hepatol 2018; 69:406-60.

24. Horan TC, Andrus M, Dudeck MA. CDC/NHSN surveillance definition of health care-associated infection and criteria for specific types of infections in the acute care setting. Am J Infect Control. 2008;36:309-32.

25. Bajaj JS. Review article: the modern management of hepatic encephalopathy. Aliment Pharmacol Ther. 2010;31:537-47.

26. Durand F, Valla D. Assessment of prognosis of cirrhosis. Semin Liver Dis. 2008;28:110-22 
27. Peng Y, Qi X, Guo X. Child-Pugh Versus MELD Score for the Assessment of Prognosis in Liver Cirrhosis: A Systematic Review and Meta-Analysis of Observational Studies. Medicine (Baltimore).2016;95:2877-2906.

28. Chen L, Lou Y, Chen Y, Yang J. Prognostic value of the neutrophil-to-lymphocyte ratio in patients with acute-on-chronic liver failure. Int J Clin Pract. 2014;68:1034-40.

29. Iliaz R, Ozpolat T, Baran B, Demir K, Kaymakoglu S, Besisik F, et al. Predicting mortality in patients with spontaneous bacterial peritonitis using routine inflammatory and biochemical markers. Eur J Gastroenterol Hepatol. 2018;30: 786-91.

30. Cai YJ, Dong JJ, Dong JZ, Yang NB, Song M, Wang YQ, et al. Neutrophil-lymphocyte ratio predicts hospital-acquired bacterial infections in decompensated cirrhosis. Clin Chim Acta. 2017:469:201-7.

31. Zhang H, Sun Q, Mao W, Fan J, Ye B. Neutrophil-to-Lymphocyte Ratio Predicts Early Mortality in Patients with HBV-Related Decompensated Cirrhosis. Gastroenterol Res Pract. 2016;2016:4394650-5.

32. Lin L, Yang F, Wang Y, Su S, Su Z, Jiang X, et al. Prognostic nomogram incorporating neutrophil-to-lymphocyte ratio for early mortality in decompensated liver cirrhosis. Int Immunopharmacol 2018; 56:58-64
33. Rice J, Dodge JL, Bambha KM, Bajaj JS, Reddy KR, Gralla J, et al. Neutrophil-to-Lymphocyte Ratio Associates Independently With Mortality in Hospitalized Patients With Cirrhosis. Clin Gastroenterol Hepatol. 2018;16:1786-91.

34. Maggi DC, Borgonovo A, Bansho ET, Soares-Silva PE, Silva TE, Colombo BS, et al. Serial assessment of hepatic encephalopathy in patients hospitalised for acute decompensation of cirrhosis. Ann Hepatol. 2019;18:331-7.

35. Yoon EL, Kim TY, Lee CH, Kim TH, Cho HC, Lee SS, et al. Long-term Prognosis of Acute-on-Chronic Liver Failure Survivors. J Clin Gastroenterol. 2019;53:134-41.

36. Cholongitas E, Senzolo M, Patch D, Kwong K, Nikolopoulou V, Leandro G, et al. Risk factor, sequential organ failure assessment and model for end-stage liver disease scores for predicting short term mortality in cirrhotic patients admitted to intensive care unit. Aliment Pharmacol Ther. 2006;23:883-93.

37. Austin MJ, Shawcross DL. Outcome of patients with cirrhosis admitted to intensive care. Curr Opin Crit Care. 2008;14:202-7.

38. Dirchwolf M, Podhorzer A, Marino M, Shulman C, Cartier M, Zunino M, et al. Immune dysfunction in cirrhosis: Distinct cytokines phenotypes according to cirrhosis severity. Cytokine. 2016;77:14-25.

39. Lin CY, Tsai IF, Ho YP, Huang CT, Lin YC, Lin CJ, et al. Endotoxemia contributes to the immune paralysis in patients with cirrhosis. J Hepatol. 2007:46:816-826. 